\title{
QED Heat Transfer
}

\author{
Thomas Prevenslik \\ QED Radiations \\ Discovery Bay, Hong Kong, China \\ nanoqed@gmail.com
}

\begin{abstract}
Classical heat transfer at the macroscale proceeds by three modes: conduction, radiation, and convection. However, at the nanoscale, heat transfer is not governed by classical physics, but rather by QM and a simplified form of QED. QM stands for quantum mechanics and QED for quantum electrodynamics. QED heat transfer is based on QM by the Planck law that requires the heat capacity of the atom to vanish under high EM confinement caused by the high surface-to-volume ratios of nanostructures thereby precluding the conservation of heat by the usual increase in temperature. EM stands for electromagnetic. Treating the nanostructure as a QM box with absorbed heat under high EM confinement, conservation proceeds by QED creating standing EM radiation that charges the nanostructure or is emitted to the surroundings. Diverse applications of QED heat transfer are described.
\end{abstract}

Keywords: heat transfer, classical physics, quantum mechanics, QED

\section{Introduction}

In classical physics, heat transfer does not depend on the size of the structure allowing conservation of EM energy to always proceed by temperature changes. QM by the Planck law [1] differs by requiring the heat capacity of the atom under EM confinement at the nanoscale to vanish, and therefore the EM energy absorbed in nanostructures cannot be conserved by the usual change in temperature. Since EM energy is absorbed almost totally in nanostructure surfaces because of their high surface-to-volume ratios, surface atoms are naturally placed under high EM confinement that by the Planck law at nanoscale wavelengths precludes the atom from having the heat capacity to change in temperature with conservation proceeding by QED inducing EM radiation that charges the nanostructure or is lost to the surroundings.

\section{Theory}

\subsection{QM Restrictions}

Classically, atoms always have heat capacity to increase in temperature upon the absorption of heat irrespective of EM confinement, but QM does not. A comparison of the thermal $k T$ energy (or the heat capacity) of the atom by classical physics and QM by the Planck law is shown in Fig. 1.

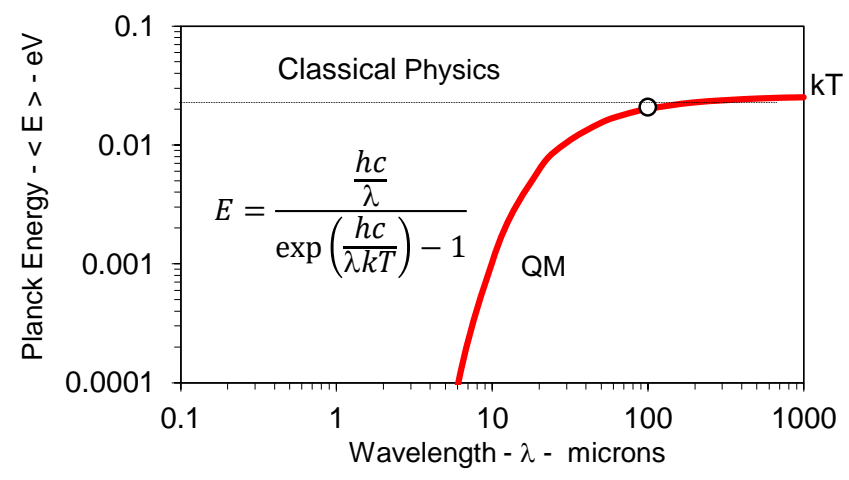

Fig. 1: Planck law of the Atom at $300 \mathrm{~K}$

$E$ is Planck energy, $h$ Planck's constant, $c$ speed of light, $k$ Boltzmann's constant, $T$ temperature, $\lambda$ wavelength. 
Fig. 1 shows QM allows the atom in macrostructures to have $k T$ energy and increase in temperature for $\lambda>100$ microns. However, $k T$ decreases for structures having $\lambda<100$ microns, while for nanostructures with $\lambda<100 \mathrm{~nm}$, the atoms have virtually no heat capacity to conserve heat by an increase in temperature.

\subsection{EM Confinement}

QED heat transfer depends on the high surface-to-volume ratios of nanostructures. A closely related mechanism is TIR standing for total internal reflection. In 1870, Tyndall showed light is trapped by TIR in the surface of a body if its RI is greater than that of the surroundings. RI stands for refractive index. However, TIR usually occurs in the surface of macroscopic bodies having low surface-to-volume ratios where heat is absorbed throughout the volume of the body.

EM confinement of nanostructures differs from TIR because their high surface-to-volume ratios requires almost all of the absorbed heat to be spontaneously deposited in their surfaces, e.g., for a nanostructure of dimension $d$ between surfaces, atoms are placed under EM confinement solely by the high concentration of surface heat, i.e., there is no physical constraint of the atoms that produces the EM confinement. Hence, EM confinement is not permanent, sustaining itself only momentarily during absorption of heat, absent absorption of heat there is no EM confinement and QED induced EM radiation is not produced.

Since QM precludes the conservation of EM energy deposited in a surface by an increase in temperature, conservation proceeds by QED inducing the creation of standing EM radiation having half-wavelength $\lambda / 2=d$, where $d$ is the dimension between opposing nanostructure surfaces. Hence, the QED induced standing wave behaves as if it were external EM radiation momentarily absorbed and frequency up or down-converted to the EM resonance of the nanostructure. Because of the nanoscale thickness $d$, the short wavelengths $\lambda$ of QED induced EM radiation are in the UV and beyond having sufficient Planck energy to ionize and charge the nanostructure, and if not, the QED radiation is emitted to the surroundings.

QED is related to complex mathematics of light -matter interaction as described [2] by Feynman, but differs here in a far simpler form, i.e., supplying heat to a QM box with sides separated by distance $d$, QED creates standing EM radiation of half-wavelength $\lambda / 2=d$. Hence, QED conserves absorbed heat under the EM confinement of the QM box by frequency up or down - conversion to the dimensions $d$ of the nanostructure, e.g., if the heat source is absorbed EM radiation, QED may blue or redshift the EM radiation. In simplified QED, the Planck energy $E$ of the EM radiation created is,

$$
E=h v, \quad v=(c / n) / \lambda, \lambda=2 d
$$

where, the speed $c$ of light in vacuum is reduced by $n$, the RI of the nanostructure.

\subsection{QED Emission}

The wavelength of EM radiation induced by QED depends on the geometry and RI of the nanostructure. Typically, $\mathrm{ZnO}$ is used for electronics and YSZ for turbine blades. YSZ is yttrium-stabilized zirconia. QED induced EM radiation is shown in Fig. 2.

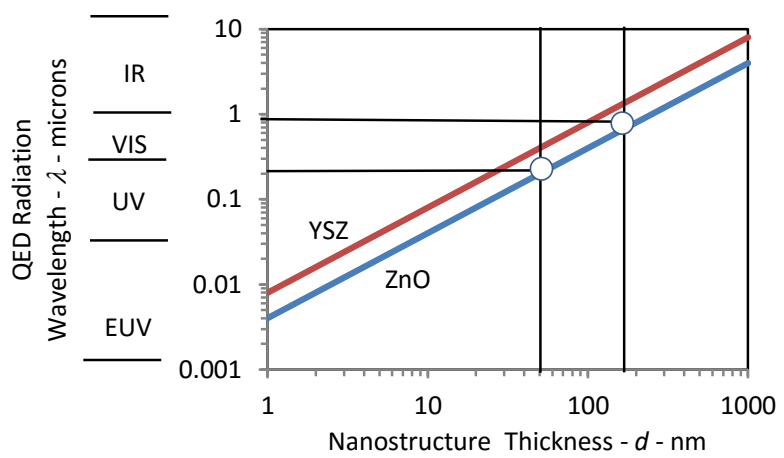

Fig. 2: QED Radiation Wavelength v. Nanostructure Thickness. 


\section{Discussion}

\subsection{Thin Films}

Cooling by QED radiation in nanoscale coatings is not new - first studied [3] by Kelemen. Since then, thin film data has been interpreted as reduced thermal conductivity because measured heat flow is lower than predicted by the Fourier equation. Because of this, the BTE was used [4] by Liu et al. to explain the reduced conductivity in thin films by the scattering of phonons. BTE stands for Boltzmann transport equation.

However, the BTE including the Fourier solutions are questionable [5] because heat balances did not include QED radiation beyond the UV lost to the surroundings, and therefore the thermal conductivity is concluded reduced from bulk. Alternatively, if the QED radiation loss is included in the balance, the conductivity remains at bulk, i.e., the heat to the film is almost totally converted to UV radiation. The exclusion of QED radiation from the heat balance is understandable [6] as frequencies beyond the UV would normally not be expected in thin film experiments.

\subsection{Turbine Blades}

Gas turbine blades are coated with TBC to insulate the blade from hot combustor gases. TBC stands for thermal boundary coating as described [7] by Lee. TBC stands for thermal boundary coating. In contrast, QED coatings [8] do not insulate the blade from high temperature, but rather cool the blade by converting combustor heat to non-thermal EM radiation that is dissipated in the surroundings. Under EM confinement in nanoscale TBCs, the heat capacity of the atom vanishes, and therefore the TBC cannot conserve heat from the combustor gases by the usual increase in temperature. Instead, conservation proceeds by the QED induced frequency up-conversion of combustor heat to non-thermal EM radiation at the EM confinement frequency of the TBC. QED cooling is passive avoiding the complexity of active fin and internal cooling by transferring the combustor heat away from the blades.

Turbine blade QED coatings differ from those in nanoelectronics because it is difficult, if not impossible to keep the coatings clean from fouling by combustor gas residues. Moreover, the application of nanoscale QED coatings of YSZ on nickel superalloys by APS and ALD is not yet demonstrated. APS stands for atmospheric plasma spray and ALD for atomic layer deposition. Conventional APS processes based on 10-100 microns particles are unlikely to produce submicron TBCs. ALD of nanoscale YSZ coatings on $\mathrm{Si}_{3} \mathrm{~N}_{4}$ are encouraging, but research to determine whether ALD is capable of producing submicron TBCs on nickel based superalloy turbine blades remains to be proven.

\subsection{EUV Lithography}

Difficulties in extending Moore's law to $13.5 \mathrm{~nm}$ may be traced to LPP lithography. LPP stands for laser produced plasma. Based on classical physics, EUV light requires LPP to ionize [9] atoms at high temperature. EUV stands for extreme ultraviolet. LPP uses high power $\mathrm{CO}_{2}$ lasers to heat solid and gas targets that produce EUV light by atomic emission. LPP systems are not only complex, but very expensive.

QED induced EUV lithography [10] based on QM offers a far simpler and inexpensive alternative in extending Moore's law, e.g., the EUV source comprises an ordinary spherical glass lens provided on the front surface with a nanoscale zinc oxide coating. A heater on the back surface supplies heat to the coating, but QM precludes any increase in coating temperature. Instead, QED converts the heat into the coating to a steady EUV light source. Lasers are not required.

The EUV wavelength is $\lambda=2 n d$, where $\mathrm{n}$ and $\mathrm{d}$ are the refractive index and thickness of the coating. For zinc oxide having $n \approx 2.5$, the QED radiation induced for coating thicknesses $\mathrm{d}<5 \mathrm{~nm}$ is in the EUV having wavelengths $<20 \mathrm{~nm}$, the degree of coherency depending on control of thickness variations. Extensions are made to mobile hand-held EUV sources for irradiating biological specimens.

\subsection{Shock Waves}

In shock waves, the work of gas compression is confined [11] almost totally in the surfaces of the nanoscale shock thickness because of its high surface-to-volume ratio. For shock thickness d, atoms between the surfaces are placed under temporary EM confinement by the high surface energy. Since the temperature cannot increase by QM, QED conserves the surface energy by creating standing EM radiation having wavelength $\lambda=2 n d$ within the shock thickness, where $n \approx 1$ for compressed gases. Because of the nanoscale shock thickness $d$, the QED radiation ionizes atoms and molecules in the shock thickness. 
By QED, the shock wave thickness $\mathrm{d}$ may be estimated by experimentally measuring the wavelength $\lambda$ of EM radiation coming off the shock wave, i.e., $d \approx \lambda / 2$, e.g., QED radiation in the UV at $\lambda=200 \mathrm{~nm}$ suggests a shock wave thickness $d=100 \mathrm{~nm}$. But QED radiation in the EUV, say at $\lambda=50 \mathrm{~nm}$ corresponding to $d=25 \mathrm{~nm}$ may be difficult as EUV is immediately absorbed in air at the shock thickness surface and cannot be measured at any distance removed from the shock wave.

\subsection{High Pressure Chemistry}

Phenomena that occur at very high pressures - thousands or tens of thousands of atmospheres - are often observed at atmospheric pressure as quantified by optical spectra. Most notable is the nitric oxide dimer reaction $2 \mathrm{NO}_{2} \rightarrow(\mathrm{NO})_{2}$ having a yield of less than $1 \%$ dimer at atmospheric pressure, but in carbon nanotubes with ends open to atmospheric surroundings the yield is almost $100 \%$. Assuming the dimers form under pressure, simple thermodynamic calculation shows that yields near $100 \%$ would only be reached at pressures of 14,000 atmospheres. MC simulations allowing the consideration of other mechanisms confirm the observed high pressure enhanced dimer yields. MC stands for Monte-Carlo molecular dynamics.

However, MC simulations based on statistical mechanics assume [12] atoms in the confined phase have classical $k T$ thermal heat capacity. Indeed, the confined phase pressure $P$ is:

$$
P=k T\left[\rho-\frac{1}{V} \ln \left\langle\exp \left(-\frac{\Delta U}{k T}\right)\right\rangle\right]
$$

where, $\rho=$ density, $V=$ volume, and $\Delta U=$ energy. In contrast, QM by the Planck law restricts the atom heat capacity depending on the EM confinement. QM stands for quantum mechanics and EM for electromagnetic. Since the $k T$ heat capacity of the atom vanishes in the confined phase, the pressure $\mathrm{P}$ also vanishes. Hence, there are no high pressures and the dimer yield in the condensed phase is enhanced by another mechanism.

In this regard, atoms under EM confinement lacking heat capacity [13] cannot conserve the energy $\Delta U$ by the usual increase in temperature. Instead, conservation proceeds by the creation of standing QED induced non-thermal EM radiation inside the nanotube at frequencies in the EUV that by photolysis explain the enhanced dimer yield.

\subsection{Near Field Enhancement}

Recently, MIT mathematicians claimed [14] that if bodies are separated by small gaps, the blackbody-limit no longer applies. Moreover, if the gaps are nanoscale, say $<100 \mathrm{~nm}$, the amount of heat transmitted between the bodies is claimed to exceed the blackbody limit by 100 to 1000 times. Since heat having wavelengths in the IR of a few microns cannot propagate across nanoscale gaps, the MIT claim is based on the assumption QM tunnels radiation across the gap from evanescent waves moving perpendicular to gap surfaces.

In near-field heat transfer, the theory [15] of evanescent waves assumes the long wavelength form of the Planck law to depend only on the temperature of the atom, i.e., for $\lambda>100$ microns in Fig. 1. However, for nanoscale gaps $g<100 \mathrm{~nm}$, atoms in the gap surfaces are under EM confinement corresponding to a standing half-wave, i.e., $\lambda / 2=g$, or $\lambda<200 \mathrm{~nm}$ where the heat capacity of the atom vanishes. Hence, the fluctuating temperatures necessary to produce the charges and currents consistent with the FDT for radiative heat transfer do not exist. Indeed, evanescent waves in near-field heat transfer do not exist in nanoscale gaps. What this means is the claim that evanescent waves enhance near-field heat transfer beyond the blackbody limit is questionable.

In the alternative, QED radiation allows heat transfer across nanoscale gaps by creating standing EM radiation that tunnels [16] heat across the nanoscale gap at the wavelength of the EM confinement is $\lambda=2 \mathrm{~g}$. However, QED predicts no enhancement of heat beyond the blackbody limit in nanoscale gaps between bodies.

\subsection{Superlens}

In conventional optics, image quality depends on the diffraction limit. Recently, transformative optics using a superlens is proposed [17] to restore image quality below the diffraction limit. Indeed, superlens experiments [18] using a $35 \mathrm{~nm}$ silver film in contact with a $40 \mathrm{~nm}$ PMMA spacer under UV illumination at $\lambda=365 \mathrm{~nm}$ showed sub-diffractionlimited imaging of etched chromium objects down to $90 \mathrm{~nm}$. PMMA stands for polymethyl methacrylate. When the 
superlens is removed, the diffraction-limited wavelength $\lambda^{*}=\lambda / n$ is controlled by the RI of PMMA, i.e., for $n=1.5, \lambda^{*}=$ $243 \mathrm{~nm}$. With the silver film, the superlens was found to resolve the average cross section of the etched chromium objects to a line width of $89 \mathrm{~nm}$.

Transformative optics depends on the evanescent enhancement across the thickness of the superlens and the condition the permittivity of the silver film and that of the adjacent PMMA are equal and of opposite sign. By QM, however, the silver film is under EM confinement across the thickness $d$ at wavelength $\lambda=2 n d$ that precludes temperature fluctuations necessary for creating evanescent waves. Similar to near-field enhancement above, evanescent waves cannot be created across the thickness of the silver film. What this means is the observed enhancement of image quality has nothing to do with evanescent waves.

Instead, sub-diffraction-limited imaging in the super lens is proposed [19] to be a natural consequence of QED induced EM radiation in the silver film. In the QED interpretation, evanescent waves are not invoked. The QED process begins with the PMMA illuminated with UV at $365 \mathrm{~nm}$ giving diffraction limited EM radiation at $\lambda^{*}=243 \mathrm{~nm}$. But the diffraction-limited EM radiation is absorbed in the silver film. Since the silver film cannot change in temperature because of QM, conservation proceeds by QED inducing the creation of EM radiation at wavelength $\lambda=2 n d$, where $\mathrm{n}$ is the RI of silver at wavelength $\lambda^{*}$ and $d$ is the silver film thickness. For $d=35 \mathrm{~nm}$ and silver at $\lambda^{*}=243 \mathrm{~nm}$ having $n=1.28$, QED allows sub-diffraction-limited line-width of $89 \mathrm{~nm}$ consistent with experimental [18] observation.

\subsection{Water Disinfection}

UVC light as a disinfectant penetrates the cell wall of an organism to scramble the genes thereby precluding reproduction. Research has shown that the optimum UV wavelength range to destroy bacteria is between $250 \mathrm{and} 270 \mathrm{~nm}$.

QED induced UV radiation using hand-held nano-coated bowls is proposed [20] to allow West Africans to disinfect water at the point of use using body heat alone - no electrical power. QED disinfection is a consequence of QM that forbids the atoms under EM confinement inherent in the nano-coating to have the heat capacity to increase in temperature. By selecting a coating thickness, say $50 \mathrm{~nm}$ of $\mathrm{ZnO}$ having $n \approx 2.5$, the heat from the hand is conserved by QED inducing conversion to UVC radiation at $\lambda \approx 254 \mathrm{~nm}$. EPA guidelines for disinfection require a UVC dose of $38 \mathrm{~mJ} / \mathrm{cm}^{2}$. With body heat of about $6 \mathrm{~mW} / \mathrm{cm}^{2}$, the hand-held drinking bowl disinfects drinking water in about 7 seconds.

Currently, LED's in the UVC are limited [21] to EQE of a few percent and not expected to impact the disinfection market until 2017/2018. LED stands for light emitting diodes and EQE for external quantum efficiency. In contrast, the QED drinking bowl has 100\% efficiency, but is limited by low body heat.

\subsection{Cosmology by Cosmic Dust}

In 1929, Edwin Hubble formulated the law that the velocity of a receding galaxy is proportional to its distance to the Earth. Hubble based his law on Doppler's Effect whereby the wavelength of light from the galaxy is redshift if the galaxy is moving away from us. Thus, by measuring the redshift of known spectral lines, Hubble claimed to know the recession velocity of the galaxy relative to the Earth.

However, cosmic dust comprising NPs of silicates permeate the vast reaches of the ISM. NP stands for nanoparticle and ISM for interstellar medium. If galaxy light is redshift upon absorption in dust which is also proportional to the distance to the Earth, velocity measurements of galaxies are no longer valid as the absorption of galaxy photons in NPs produces the redshift $\mathrm{Z}$,

$$
\mathrm{Z}=\left(\lambda_{o}-\lambda\right) / \lambda
$$

where, $\lambda$ is the wavelength of the galaxy photon which is redshift in the NP to wavelength to $\lambda_{o}=2 n d$.

Cosmological consequences are enormous as astronomers [22] based on the redshift of SN light have taken Hubble's law as proof the Universe is not only expanding, but the expansion is accelerating. SN stands for Supernovae. Redshift in dust means Universe expansion need not be occurring, let alone accelerating, thereby allowing the outstanding problems in cosmology to be simply resolved by Newtonian mechanics.

Regardless, the Hubble measurements of redshift may need correction for cosmic dust. In this regard, the Hubble redshift $\mathrm{Z}_{\mathrm{Hubble}}$ by the Doppler Effect gives the same $\mathrm{Z}$ for ALL galaxy photon wavelengths while QED induced redshift in dust depends on wavelength. A reasonable dust correction to Hubble's law depends on the difference between the 
shortest and longest wavelength galaxy photons, e.g., Ly $\alpha$ and H $\alpha$. Therefore, valid Hubble redshift from measured ZMeas is proposed [23] corrected using measured $\mathrm{Z}$ for individual Ly $\alpha$ and $\mathrm{H} \alpha$ lines,

$$
\mathrm{Z}_{\text {Hubble }}=\mathrm{Z}_{\text {Meas }}-\left(\mathrm{Z}_{\mathrm{Ly} \alpha}-\mathrm{Z}_{\mathrm{H} \alpha}\right)
$$

where Ly $\alpha$ and $\mathrm{H} \alpha$ stand for the $\alpha$ lines of Lyman and hydrogen.

\subsection{Exoplanets}

Discovery of exoplanets in far reaches of galactic space is a difficult task because of inherently faint images. Similar to the problems of black hole mass and galaxy rotation, proof of discovery is based on high-precision spectroscopy [24] to measure variations in light intensity as the planet spins. When observing a spinning exoplanet, half of the planet rotates away from Earth, while the other half rotates toward Earth. The spectrum of the side spinning away from us will show redshift, whereas the side spinning toward us will show a spectral blueshift.

The discovery by Oppenheimer et al. [25] of 4 planets orbiting the distant star HR8799 supported the theory that exoplanets surround other stars in the Universe is consistent with the planets that orbit our Sun. IR spectroscopic measurements of the suspected planets identified ammonia from 1450 to $1550 \mathrm{~nm}$, acetylene from 1500 to $1550 \mathrm{~nm}$, methane above $1650 \mathrm{~nm}$, and possibly carbon dioxide from 1560 to $1630 \mathrm{~nm}$, all of which are commonly observed in the atmospheres of planets in our solar system. However, molecular lines of carbon dioxide and methane should have been found in all 4 planets, but were only observed for 2 planets. In one planet, a weak carbon dioxide line initially not present appeared 4 months later. What this means is the spectra of HR8799 system are not likely produced by molecules.

QED redshift suggests the molecules thought measured by IR spectroscopy are in fact redshifted Ly $\alpha$ photons having a wavelength $\lambda_{o}$ depending on the NP properties. Indeed, NPs may produce near IR spectra similar to that of common molecules at high redshifts $\mathrm{Z}$ of Ly $\alpha$ photons. From Equation 3, the QED redshift wavelength $\lambda_{\mathrm{o}}=(\mathrm{Z}+1) \lambda$. A silicate NP having diameter $d \approx 500$ microns and $n \approx 1.5$ redshifts galaxy photons to a wavelength $\lambda_{\mathrm{o}}=2 \mathrm{nd} \approx 1500 \mathrm{~nm}$. For Ly $\alpha$ photons having $\lambda=121.7 \mathrm{~nm}$, the redshift $\mathrm{Z}=11.3$. Hence, the NP redshift to $1500 \mathrm{~nm}$ could be mistaken as the ammonia line at $1450-1550 \mathrm{~nm}$. Consistent with observation, the IR spectra has nothing to do with any molecular species, but most likely depends on cosmic dust NPs. Hence, redshifted Ly $\alpha$ radiation in dust to the near IR could be interpreted as proof of exoplanet discovery - when in fact the exoplanet may not even exist.

\subsection{ISM Infrared Spectra}

Dust NPs are thought to emit EM radiation by thermal emission at near to far IR wavelengths from non-equilibrium heating by single galaxy photons. In 1976, Purcell [26] showed single-photon heating produces a temperature spike of 40 $\mathrm{K}$ in very small NPs $(d<0.005$ microns) so that much of the absorbed energy will be radiated in the far IR near 60 microns. Today, Li and Draine [27] also propose NPs heated by single galaxy photons are the source of IR spectra.

However, the notion that NPs heat-up upon absorption of single galaxy photons is based on classical physics that allows the atom to have heat capacity. Contrarily, QM denies NP atoms under EM confinement the heat capacity to conserve the absorbed galaxy photon by a spike in temperature. Instead, QED produces IR in the ISM by redshift of Lya photons as described above for exoplanets without increasing the NP temperature.

\section{Conclusions}

QED heat transfer finding basis in QM is proposed at the nanoscale as the mode of heat transfer alongside the classical modes of conduction, radiation, and convection at the macroscale.

At the macroscale, conduction is thought to be the most efficient mode of heat transfer. But at the nanoscale, the lack of heat capacity of the atom by QM precludes the temperature 'changes necessary for thermal conduction, radiation, and convection. Unlike the macroscale, the Fourier heat equation is no longer applicable at the nanoscale.

Heat transfer at the macroscale conserves heat by changes in temperature, but at the nanoscale heat is conserved by creating charge or is lost to the surroundings.

Diverse applications of QED heat transfer presented based on QM suggest that classical physics at the nanoscale should henceforth be discontinued. 


\section{References}

[1] M. Planck, "On the Theory of the Energy Distribution Law of the Normal Spectrum," Verhandl. Dtsch. Phys. Ges., vol. 2, pp. 237, 1900.

[2] R. Feynman, QED: The Strange Theory of Light and Matter, Princeton University Press, 1985.

[3] F. Kelemen, "Pulse Method for the Measurement of the Thermal Conductivity of Thin Films," Thin Solid Films, vol. 36, pp. 199-203, 1976.

[4] W. Liu, Y. Yang, and M. Asheghi, "Thermal and Electrical Characterization and Modelling of Thin Copper Layers," in Proceedings of Intersociety Conference on Thermal and Thermomechanical Phenomena in Electronic Systems, ITHERM, 2005.

[5] T. Prevenslik, "Heat Transfer in Thin Films," in Proceedings of the Third Inter. Conf. on Quantum, Nano and Micro Technologies, ICQNM, Cancun, 2009.

[6] T. Prevenslik. (2010-2016). QED Theory and Applications [Online]. Available: http://www.nanoqed.org

[7] K. N. Lee, "Protective Coatings for Gas Turbines" in The Gas Turbine Handbook, R. A. Dennis, Ed. Section 4.4.2, NETL, 2006.

[8] T. Prevenslik, "QED Cooling of Gas Turbine Blades," in Proceedings of ASME Turbo Expo 2014: Turbine Technical Conference and Exposition, Düsseldorf, Germany, 2014.

[9] B. A.M. Hansson, et al., "LPP EUV Source Development for HVM," in Proceedings of Emerging Lithographic Technologies X of SPIE, vol. 6151, 2006.

[10] T. Prevenslik. (2014, May 5). Lithography by QED induced EUV Light Sources [Online]. Available: http://www.prlog.org/12317992-lithography-by-qed-induced-euv-light-sources.html

[11] T. Prevenslik. (2015, August 6). Shock Waves produce QED radiation - not high Temperatures! [Online]. Available: http://www.prlog.org/12474176-shock-waves-produce-qed-radiation-not-high-temperatures.html

[12] Y. Long, et al., "On the molecular origin of high-pressure effects in nanoconfinement: The role of surface chemistry and roughness," J. Chem. Phys., vol. 139, 144701, 2013.

[13] T. Prevenslik. (2015, October 13). Chemistry in confined phases by Quantum Mechanics [Online]. Available: http://www.prlog.org/12501177-chemistry-in-confined-phases-by-quantum-mechanics.html

[14] O. D. Miller, S. G, Johnson, and A. W. Rodriguez, "Shape-Independent Limits to Near-Field Radiative Heat Transfer," PRL. 115, 204302, 2015.

[15] K. Joulain, et al., "Surface electromagnetic waves thermally excited: Radiative heat transfer, coherence properties and Casimir forces revisited in the near field," Surf. Sci. Rep., vol. 57, pp. 59, 2005.

[16] T. Prevenslik, "Near Field Radiation by Quantum Mechanics," in Proceedings of the ASME $20134^{\text {th }}$ Micro/Nanoscale Heat \& Mass Transfer International Conference MNHMT2013-22026, Hong Kong, China, 2013. [Online]. Available: http://www.prlog.org/12517435-evanescent-waves-do-not-exist-in-near-field-heat-transfer.html

[17] J. B. Pendry, "Controlling Light on the Nanoscale," Progress in Electromagnetics Research, vol. 147, 117, 2015.

[18] D. O. S. Melville and R. J. Blaikie, "Super-resolution imaging through a planar silver layer," Optics Express, vol. 13, 6, 2127, 2002.

[19] T. Prevenslik. (2015, November 24). Super image quality by transformative optics or QED? [Online]. Available: http://www.prlog.org/12512936-super-image-quality-by-transformative-optics-or-qed.html

[20] T. Prevenslik, "QED Disinfection of Drinking Water in China," in Proceedings of Inter. Conf. on Water Resource and Environment (WRE2015), Beijing, China, 2015.

[21] P. Mukish, UV LED - Technology, Manufacturing and Application Trends in disinfection, Yole Development, 2015.

[22] A. G. Riess, et al., "Type Ia Supernova Discoveries at Z $>1$ from the Hubble Space Telescope Evidence for Past Deceleration and constraints on Dark Energy Evolution," ApJ, vol. 607, 665, 2005.

[23] T. V. Prevenslik, "Cosmic Dust and Cosmology," in Proceedings of APRIM 2014: Galaxies, AGN and Cosmology Daejon, Korea, 2015.

[24] I. A. G. Snellen, et al. "Fast Spin of the Young Extrasolar Planet Beta Pictoris b," Nature, April 30, 2014.

[25] B. R. Oppenheimer, et al., "Reconnaissance of the HR 8799 exosolar system I: Near IR spectroscopy," arXiv:1303.2627v1 [astro-ph.EP].

[26] E. M. Purcell, "Temperature fluctuations in very small interstellar grains,” Astrophys. J. , vol. 206, pp. 685, 1976.

[27] A. Li, and B. T. Draine, "Infrared Emission from Interstellar Dust. III. The Small Magellanic Cloud," AsJ, vol. 576, pp. 762-772, 2002. 